\title{
Mikis Theodorakis : la rencontre éternelle de la Poésie et de la Musique
}

\author{
Kalliopi STIGKA (Athènes) ${ }^{1}$
}

\section{Summary}

Poetry and Music. Music and Poetry. Two expressions of one unique sensitivity, that of the great contemporary Greek composer, politician and philosopher, Mikis Theodorakis (born 1925). The union of Poetry and Music came about when Mikis Theodorakis put to music the poem of an unknown

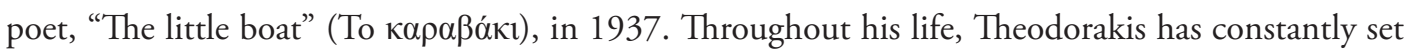
to music the works of the greatest Greek and foreign poets, such as Odysseus Elytis, Yannis Ritsos and Pablo Neruda. Through their poetry, Theodorakis has been able to sing a mother's tender love as well as the sadness of a solitary man, the darkness of a concentration camp or the serenity of a Cycladic landscape.

Yet, there are times when the poetic words of other poets do not suffice and so he, himself becomes the poet. When? Why? What are his motifs and his intentions? The aim of this article is on the one hand to highlight the poetic creation of Mikis Theodorakis - an expression of eternal humanism through an anthology of poems written in Greek during periods of extreme violence. On the other hand, to reveal the new musical forms which were born of the union of Poetry and Music and to stress the role of the performers-interpreters in the transmission of theodorakian music throughout the world.

Depuis plusieurs années notre objectif est d'explorer l'œuvre particulièrement riche et variée de Mikis Theodorakis et de la transmettre. De la chanson à la musique symphonique et méta-symphonique, du poème au roman autobiographique et à l'essai politico-philosophique, l'œuvre de cette personnalité emblématique de la Grèce contemporaine est une source inépuisable de nouveauté, d'inspiration et de force.

Par notre communication - prononcée dans le cadre du Colloque International Chanter les poètes. Poésique 18 qui était organisé par l'Institut des langues et cultures d'Europe, d'Amérique, d'Afrique, d'Asie et d'Australie et qui, à notre sens, avait un caractère davantage littéraire que musical - nous avons souhaité mettre en exergue l'œuvre poétique de Mikis Theodorakis qui dans sa plus grande partie a donné naissance à de nombreuses œuvres 
musicales. Cet article qui constitue une version enrichie et légèrement modifiée de notre communication ne prétend pas être une analyse poético-musicale au sens strict du terme. Son but est, avant tout, en présentant l'œuvre poétique théodorakienne, d'y attirer l'attention des chercheurs non-hellénophones pour qui l'accès aux textes est souvent limité, faute de traductions, et de provoquer de nouvelles recherches de cette ouvre dans son ensemble. Les processus compositionnels qui, à travers la rencontre de la Poésie et de la Musique, ont donné naissance à de nouvelles formes musicales, ainsi que le rôle important de l'interprète à la transmission de cette œuvre monumentale seront également mentionnés.

Etant particulièrement sensible à la notion de ‘ censure > toujours présente, hélas, de nos jours - sous des formes plus discrètes -, nous avons choisi des poèmes/chansons - parfois censurés - créés lors des périodes violentes de la vie de Mikis Theodorakis. Par ailleurs, comme la vie de Theodorakis-créateur est intimement liée à la vie de Theodorakis-combattant-penseur-homme politique, ce périple entre ses mots et ses mélodies ne peut se faire qu'en retraçant le cheminement de l'histoire grecque contemporaine.

«Parce que j'ai trois vies...»

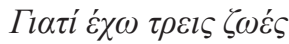

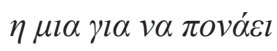 $\eta \dot{\alpha} \lambda \lambda \eta \gamma \iota \alpha$ va $\theta \dot{\varepsilon} \lambda \varepsilon l$

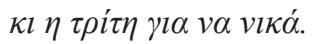

Parce que j'ai trois vies

Une pour souffrir

Une autre pour désirer

Et une troisième pour vaincre.

(Theodorakis 2000, 63)

Existe-t-il une meilleure façon pour décrire de manière concise la vie si pleine, si intense, si bouleversée et si bouleversante de Mikis Theodorakis que ces trois vers issus de son poème

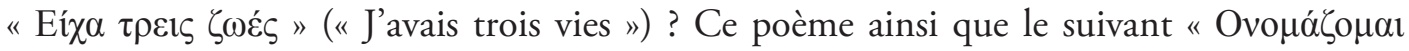

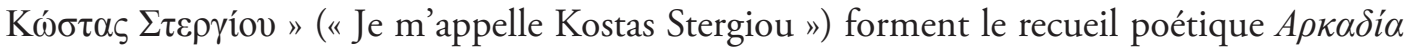
$X($ Arcadie $X)$ écrit le 21 septembre 1969 à Zatouna en Arcadie, village où il était exilé avec sa famille par les Colonels. Ce jour-là, le lieutenant de Police Kostas Stergiou, célèbre pour sa cruauté, a été envoyé par la Junte, pour ramener l'épouse et les enfants de Theodorakis à Athènes, après leur avoir imposé une humiliante fouille au corps. Ce jour-là également, après deux ans d'emprisonnement, de tortures et d'exil, Theodorakis se retrouve seul, isolé dans la maison de Zatouna. Les mots seront pour lui, une fois de plus, une arme silencieuse qui l'aideront à exprimer sa rage, à calmer son désespoir, à retrouver sa force intérieure inépuisable. 
Pendant quatre-vingt-quinze ans, Mikis Theodorakis a vécu et vit encore plusieurs vies. La première - "Une [vie] pour souffrir " - a été une vie pleine de combats, de douleurs, de souffrance, de mort. Comment oublier son combat pendant la Résistance ? Comment oublier la douleur et les tortures inimaginables infligées par ses bourreaux lors de son exil sur l'île de Makronissos², pendant la Guerre Civile Grecque (1946-1949) ? Comment oublier les souffrances éprouvées en entendant les cris de ses codétenus torturés sur la terrasse du siège de la Police sous la Dictature des Colonels (1967-1974) ? Comment oublier la mort de ses camarades? Comment oublier également les assassinats de Grigoris Lamprakis, homme pacifiste, député de l'Union Démocratique de Gauche (EDA) en 1963 ou celui du jeune étudiant Sotiris Petroulas tué lors d'une manifestation, en 1965 ?

La seconde vie - "Une autre [vie] pour désirer " - au cours des années cinquante sera remplie d'espoir, de désirs, d'enthousiasme créatif, lorsqu'il part en France accompagné de son épouse Myrto Altinoglou. C'est au Conservatoire de Paris qu'il complètera ses études et suivra les classes d'Olivier Messiaen et d'Eugène Bigot. C'est là également qu'il débutera sa carrière de compositeur de musique savante en créant, entre autres, des œuvres de musique de chambre, des musiques de film, des musiques de ballet; une carrière brillante qui le conduira au Prix d'Or du Concours de Composition organisé par le Festival International de Jeunesse de Moscou (1957), au Prix Copley (1959) ainsi qu'au Covent Garden (19581960). Mais ce monde élitiste ne le comblera pas pleinement.

De retour en Grèce, au début des années soixante, il constate une grande instabilité politique, un anticommunisme extrême, le chômage, l'analphabétisme et une grande pauvreté. Pour lutter contre ce chaos, s'inspirant de la révolution culturelle soviétique et persuadé que "l'instruction du peuple [...] est un des éléments qui contribuent à sa libération " (Stiga 2006, 1113), il posera les bases du "mouvement de la culture combattante ", lié aux forces politiques progressistes de l'époque. Le but de ce mouvement est, non seulement la démocratisation de la vie sociale, mais aussi la renaissance puissante du peuple grec. Afin d'atteindre cet objectif Mikis Theodorakis fait appel à l'Art qu'il considère comme « un des facteurs qui peut déterminer le peuple et le transformer en puissance historique [...]. Mais un art qui parle au peuple et non pas un art que le créateur garde pour lui-même [...]" (Stiga 2006, 1097). En réalité, il souhaite rendre accessible à tous l'Art, jusqu’alors destiné uniquement à l'élite grecque et non pas au peuple, contribuant ainsi au rapprochement des classes sociales. C'est à ce moment-là qu'il réalisera son objectif : créer une musique non classée socialement, une "musique pour les masses ». La "musique pour les masses » est née du " mariage de la musique traditionnelle et populaire grecque et de la poésie contemporaine néo-hellénique » (Theodorakis 1972, 22). Mais comme l'explique Kostas Serezis :

[...] le terme ‘ masse ` ne désigne pas cette fois-ci un public impersonnel qui se comporte, plus ou moins, selon les règles de la psychologie des masses. Il désigne un grand nombre de personnes qui suivent fascinés un phénomène intellectuel, comme d'autres suivent, souvent par intérêt personnel, un leader politique dont l'influence est, la plupart du temps, éphémère. Mikis Theodorakis guide par sa musique, aide les gens à 
se souvenir et non pas à oublier. Il (grimpe la pente > et emmène les autres avec lui.

(Serezis 2002, 11-12)

Comme cette initiative de Theodorakis aura lieu lors d'une période où le peuple grec a un fort besoin d'exprimer ses inquiétudes et ses chagrins et de lutter pour une vie meilleure tout en étant soutenu et guidé par un leader charismatique, la réussite ne pouvait qu'être certaine. C'est également dans les premières années du Nouveau Régime - après la chute de la dictature en juillet 1974 - que le peuple grec enfin libéré, après sept ans de censures et d'interdictions, chantera avec passion les œuvres de Theodorakis interdites pendant le septennat. Ces trente dernières années, il savoure en vainqueur une troisième vie - «Et une troisième [vie] pour vaincre " - qui lui a apporté la reconnaissance mondiale. Dans le même temps, il continue à créer et à participer activement à la vie sociale et politique de la Grèce, malgré son âge avancé.

\section{La création}

Lélément qui lie ces trois vies de Mikis Theodorakis, ce sont les mots. Ses écrits qui font preuve d'une personnalité impétueuse et extraordinairement polyvalente, peuvent être classés en trois grandes catégories : les textes sur la musique et la culture - comme $H$ Movølkń

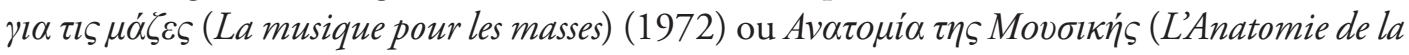

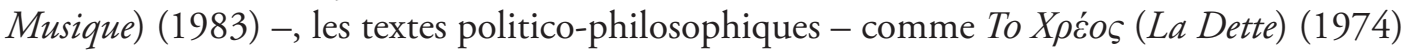

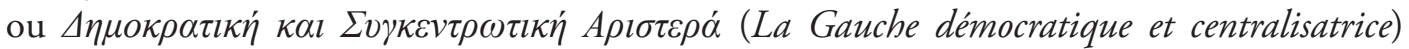
(1976) -, et les textes littéraires. Dans cette dernière catégorie figurent son roman autobio-

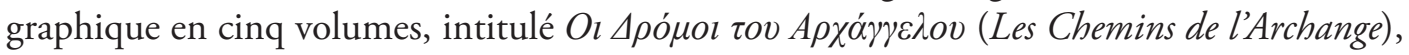
écrit et publié entre 1986 et 1995, et ses recueils poétiques.

La première rencontre de la Poésie et de la Musique a lieu en 1937, quand Mikis Theo-

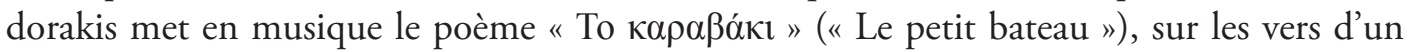
poète inconnu. Depuis ce jour, il ne cesse de mettre en musique les œuvres des grands poètes

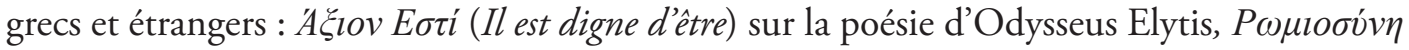

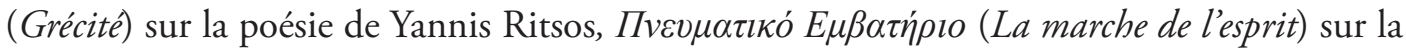
poésie d'Angélos Sikélianos, Canto General sur la poésie de Pablo Neruda, etc. Grâce à la parole poétique d'Odysseus Elytis, de Yannis Ritsos, d'Angélos Sikelianos, de Georges Séféris, de Paul Eluard, de Pablo Neruda, d'Albert Camus, de Léopold Sédar Senghor, etc., Theodorakis a réussi à chanter aussi bien l'amour tendre d'une mère pour son fils que l'amour fou d'une femme pour son amant, le chagrin d'un homme seul autant que la lamentation d'une mère devant le corps inanimé de son fils assassiné, l'obscurité d'un camp de concentration comme la sérénité du paysage cycladique sous la lumière du soleil grec, mais avant tout Theodorakis a uni sa voix à celle de l'homme qui lutte pour la Paix, la Liberté et la Démocratie et a toujours crié fort : «Unissez-vous!» 
La mise en musique de la poésie a donné naissance à un grand nombre de chansons et de cycles de chansons mais également à une " tragédie populaire musicale contemporaine "

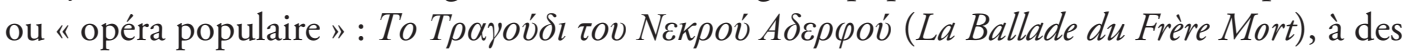
œuvres méta-symphoniques - c'est-à-dire à des chansons-fleuves, comme $H A \delta \varepsilon \rho \varphi \eta ் ~ \mu \alpha \varsigma$

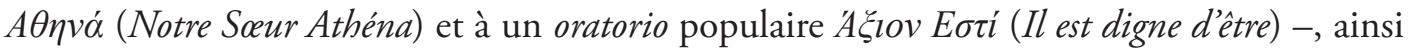

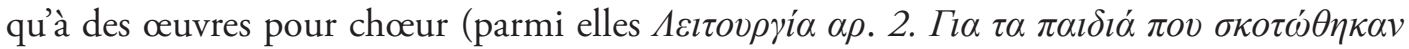

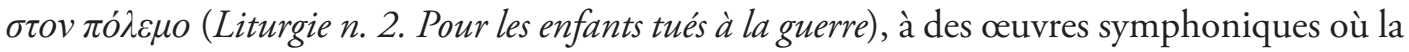

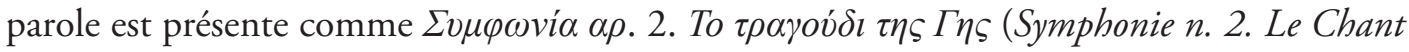

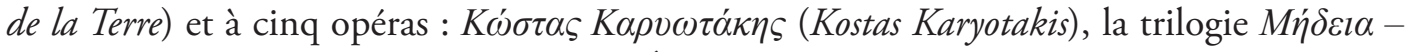

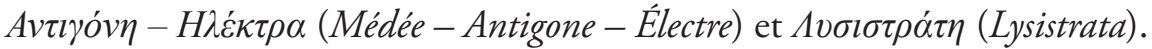

Il choisit les poèmes qu'il va mettre en musique selon leur originalité, le message qu'ils véhiculent et les sentiments qu'ils éveillent dans l'âme du peuple. Le compositeur lui-même témoigne :

Il y a un élément déterminant qui me conduit finalement vers tel ou tel chemin de composition : la sensibilité du public. Il y a un climat, une attente dans le peuple que tu peux capter seulement avec les antennes de ton ‘ âme `. Si les réflexes de ton âme sont corrects, alors tu peux capter cette ambiance générale qui couvre cette attente sous la surface des événements. (Holst 1980, 258)

Cependant, parfois la parole poétique des autres est insuffisante pour exprimer complètement son état d'esprit et c'est alors qu'il devient poète lui-même. Quand ? Pourquoi ? Quels sont ses motifs et ses intentions?

Son aventure avec les mots commence dès son adolescence. Ainsi, l'édition de son premier recueil poétique intitulé $\Sigma I A O(S I A O)$ qui date de 1942, porte la date antérieure de 1939, puisque toute publication était interdite par les autorités sous l'Occupation (Theodorakis 1986, 199). Selon l'écrivain et archéologue Grigoris Konstantinopoulos, avec les poèmes de

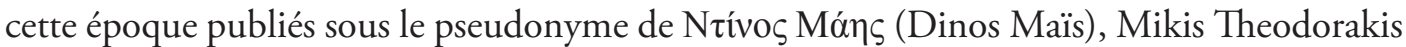
"a dessiné avec vivacité [son] âme juvénile, inquiète et pure " (Theodorakis 1986, 208),

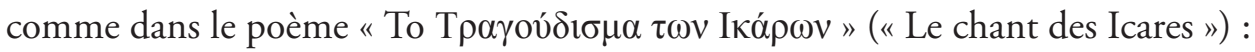

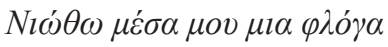

$v \alpha \mu \varepsilon \lambda \imath \omega ́ v \varepsilon l \kappa \alpha l v \alpha \mu \varepsilon \sigma \omega ́ v \varepsilon l !$

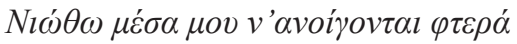

$\kappa \alpha l v \alpha \mu \varepsilon v \psi \omega ́ v o v v$

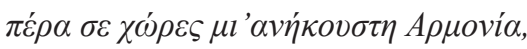

$\kappa \alpha \iota \mu \varepsilon \mu \iota^{\prime} \alpha \theta \dot{\rho} \rho \eta \tau \eta O \mu о \rho \varphi \iota \dot{\alpha} !$
}

Je sens en moi une flamme

qui me fait fondre et qui me sauve!

Je sens en moi des ailes qui s'ouvrent 
et qui me soulèvent

au loin au dessus des pays d'une Harmonie inaudible,

et d'une Beauté invisible!

(Theodorakis 1986, 204, v. 1-6)

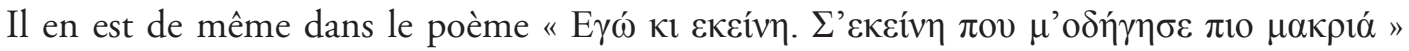
("Moi et elle. A celle qui m’a mené plus loin ») où il évoque le premier amour et les rêves des jeunes amoureux :

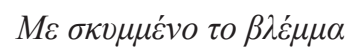

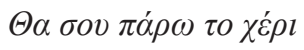

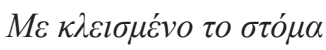

$\Theta \alpha \sigma o v \pi \omega^{\prime} \ll \sigma^{\prime} \alpha \gamma \alpha \pi \omega^{\prime} ! »$

Bं́ $\mu \alpha \beta \dot{\eta} \mu \alpha \alpha \nu \tau \dot{\alpha} \mu \alpha$

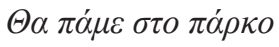

Kl $\alpha \gamma \kappa \alpha \lambda \imath \alpha \sigma \mu \varepsilon \dot{v o l}$

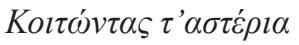

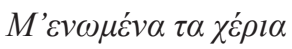

$\Theta \varepsilon v^{\prime} \alpha \kappa o v ́ \mu \varepsilon \beta$ вовоí

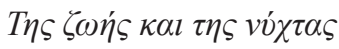

$T \eta v \omega \delta \eta \dot{\eta} \mu \varepsilon \tau o v i \delta$ io

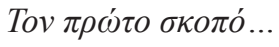

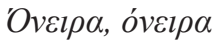

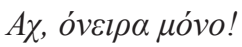

Avec le regard penché

Je prendrai ta main

Avec la bouche fermée

Je te dirai “je t'aime!"

Pas à pas ensemble

Nous irons au parc

Et enlacés

En regardant les étoiles

Avec les mains unies

Nous écouterons en silence

Le chant de la vie et de la nuit

Sur la même mélodie...

Rêves, rêves,

Ah! seulement des rêves !

(Theodorakis 2000, 69, v. 5-19) 
Cependant, la Résistance et la Guerre Civile l'ont profondément affecté. Et malgré sa " période française " (1953-1959), fructueuse et heureuse, Theodorakis une dizaine d'années plus tard souffre encore des blessures de la Guerre Civile. La mort de ses camarades, la haine que cette guerre fratricide a semée dans la société grecque hantent son esprit et il n'a qu'un seul souhait : lutter pour l'unité des Grecs. Comment va-t-il procéder ? Avec ses armes : les mots et les mélodies.

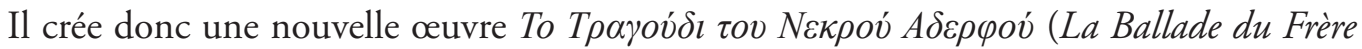
Mort) (1960-1963), inaugurant en même temps un nouveau genre musical : " la tragédie musicale populaire contemporaine " ou " opéra populaire ». Il s'agit d'un genre musical d'inspiration théodorakienne qui se situe entre le drame antique et la tragédie lyrique contemporaine. Dans La Ballade du Frère Mort, il ose évoquer le sujet le plus controversé de l'histoire contemporaine grecque, celui de la Guerre Civile. Il écrit un scénario complet, les dialogues, les vers des chansons et la musique et propose également des idées de mise en scène pour cette œuvre qu'il imagine comme : "un mouvement continu et compact de musique et de danse " (Theodorakis 1997a, 88). L'œuvre, par le sujet qu'il traite ainsi que par sa mise en musique originale, provoque au moment de sa création des réactions intenses aussi bien dans les milieux musicaux grecs que chez le public.

Parmi les douze chansons de l'œuvre, les chansons « $\mathrm{H} \alpha \lambda v \sigma i ́ \delta \alpha$ " (" La chaîne »), «'Ev $\alpha$

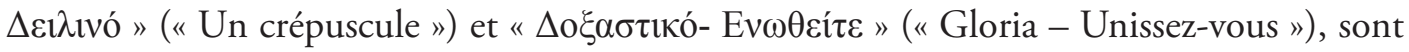
particulièrement importantes pour leur contenu sémantique ainsi que pour leur mise en musique. "La chaîne ", la sixième chanson de l'œuvre, a été censurée pendant les années soixante ainsi que pendant la Dictature des Colonels car c'est un appel à la lutte et à la révolte :

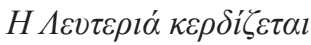

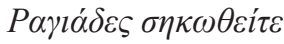

La Liberté se gagne

Esclaves levez-vous

(Theodorakis 1997a, 90, v.25-27)

Elle fait allusion aux Grecs de la Résistance, quand ils étaient tous unis - " amis d'hier, ennemis d'aujourd'hui " (Theodorakis 1974, 369) - et luttaient contre l'occupant étranger. Mais pour que les luttes soient réussies, l'unité du peuple et le travail collectif sont indispensables :

\footnotetext{
$\operatorname{T\eta v} \alpha \lambda v \sigma i \delta \alpha \pi o v \mu \imath \lambda \dot{\alpha}$

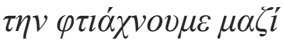

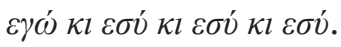


La chaîne qui parle

nous la faisons ensemble

moi et toi et toi et toi.

(Theodorakis 1997a, 90, v.22-24)

Dans la chanson "Un crépuscule " qui est la septième chanson de l'œuvre, le chagrin de la mère endeuillée par la disparition de son fils est mis en lumière. La passion de ce jeune homme courageux est comparée à la Passion du Christ :

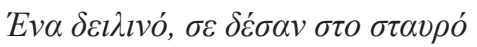

Un crépuscule, ils t’ont attaché sur la croix.

(Theodorakis 1997a, 91, v.1)

Puis, la mère parle de son fils et sa lamentation renvoie à celle de la Vierge-Marie devant le corps martyrisé de son fils :

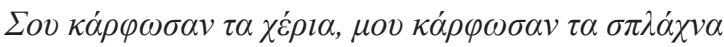

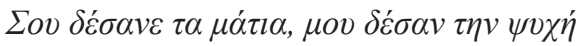

'Ev $\delta \varepsilon \imath \lambda \imath v o ́, \mu \varepsilon \tau \sigma \alpha \dot{\alpha} \kappa l \sigma \alpha v \sigma \tau \alpha \delta v o ́$.

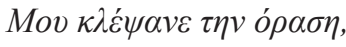

$\mu o v \pi \dot{\rho} \rho \alpha v \tau \eta v \alpha \varphi \eta \dot{~} \mu o v$

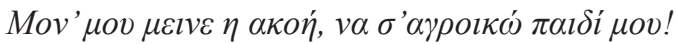

Ils t'ont cloué les mains, ils m’ont cloué les entrailles.

Ils t'ont bandé les yeux, ils m'ont bandé l'âme.

Un crépuscule, ils m’ont brisé en deux.

Ils m’ont volé la vue,

ils m'ont pris le toucher.

Il ne m'est resté que l'ouïe pour t'entendre, ô mon enfant !

(Theodorakis 1997a, 91, v.2-7)

Les thèmes de la Passion, de la Crucifixion et de la Résurrection du Christ sont une source d'inspiration constante pour les poètes et écrivains grecs néo-hellènes de la Gauche,

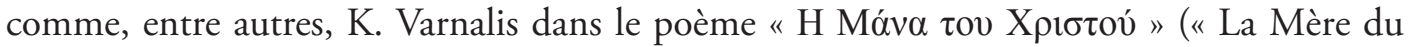

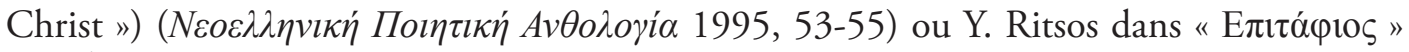
("L'Épitaphe») (Beaton 1996, 158 et 206).

En ce qui concerne la dernière chanson de l'œuvre "Gloria - Unissez-vous ", c'est un hymne de réconciliation : 
Les éléments de la nature s'unissent :

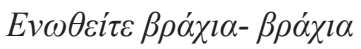

Unissez-vous, rochers aux rochers.

(Theodorakis 1997a, 93, v.1)

Les mains des gens s'unissent :

$E v \omega \theta \varepsilon i \tau \varepsilon \chi \chi^{\prime} \rho l \alpha \chi \varepsilon^{\prime} \rho l \alpha$

Vos mains dans les mains

(Theodorakis 1997a, 93, v.2)

Et comme toute réconciliation, celle-ci doit être célébrée :

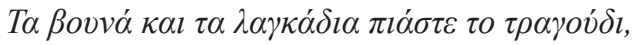

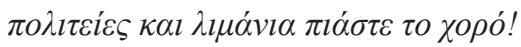

Montagnes et vallées, mettez-vous à chanter

Villes et ports, entrez dans la danse !

(Theodorakis 1997a, 93, v.3-4)

Elle est enfin couronnée par le mariage du Soleil et de la Fleur de Lilas ! Il s’agit du « jour plein de gloire " (v.13) où avec l'aide de la Vierge-Marie, on lutte pour la délivrance ${ }^{3}$ :

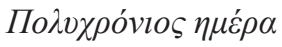

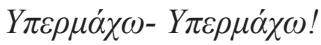

Jour plein de gloire

Ypermacho-Ypermacho!

(Theodorakis 1997a, 91, v.13-14)

Il faut noter que la fleur de lilas est le symbole de Pâques, donc de la Résurrection et de la délivrance de tous les maux.

Quant à la mise en musique de ces trois chansons, l'influence de la musique byzantine (répétition de la même note), de la musique démotique (utilisation fréquente des rythmes impairs, par ex. du zeibékikon en 9/8) et des rébétikas (utilisation des makams) est apparente. Ainsi le compositeur fait appel aux " racines musicales " des Grecs, ce qui rend les chansons plus facilement mémorisables et les messages véhiculés plus rapidement adoptés. 
Mais le "mouvement politico-culturel de renaissance " qui a donné naissance à cette œuvre parmi bien d'autres est stoppé brutalement en 1967 par la Junte des Colonels. Theodorakis est le premier intellectuel à lancer un appel à la résistance quelques jours seulement après le putsch et ne cessera jamais d'inciter le peuple à la révolte durant cet obscur septennat.

Dans La Ballade du Frère Mort, une seule chanson a connu la censure. Mais, pendant la dictature, c'est l'ensemble de la création théodorakienne qui est interdite. Mikis Theodorakis n'arrête pas pour autant de créer. Écrire est alors pour lui un véritable acte de résistance. Cela l'aide à rester en vie lors des interminables périodes d'arrestation, d'emprisonnement et d'exil. Ses vers deviennent une arme de dénonciation, de protestation, de délivrance, d'appel à la lutte et à l'union ou tout simplement un moyen de rendre hommage à ceux qui donnent leur vie pour la Paix et la Démocratie.

Nous tenons à nous référer aux cycles de chansons $O$ 'H

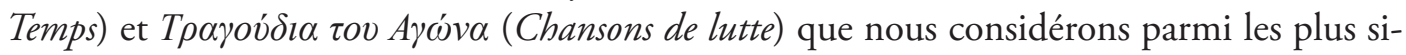
gnificatifs de cette période. Les deux sont créés à l'étranger par des chanteurs grecs ou étrangers qui luttent pour la restauration de la démocratie en Grèce. Evoquant les conditions dans lesquelles l'œuvre poétique Le Soleil et le Temps est née, Mikis Theodorakis explique dans

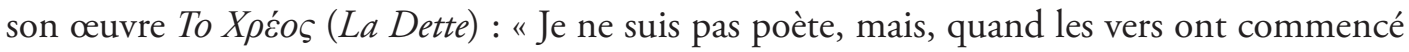
à marteler mon esprit, j'ai senti à quel point les mots peuvent se vêtir de sang ; à quel point ils peuvent me délivrer. " (Theodorakis 1974, 258)

Ainsi à Athènes en septembre 1967, sous la Junte, au siège de la Police, dans la cellule n.4, trente-deux poèmes aux vers irréguliers voient le jour ; des mots ardents jaillissent d'un esprit oppressé :

\section{$T \alpha \kappa \varepsilon \lambda l \alpha \dot{\alpha} \alpha v \alpha \sigma \alpha i v o v v$

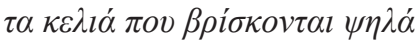

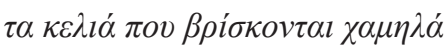

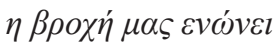

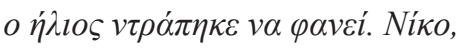

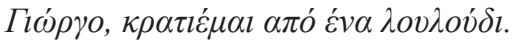

Les cellules respirent

les cellules situées au-dessus

les cellules situées au-dessous

la pluie nous réunit

le soleil n'a pas osé apparaître. Nikos,

Georges, je n'ai qu'une fleur pour me retenir

(Theodorakis 1997a, 186)

Les poèmes dans leur ensemble décrivent le ‘ combat ` entre le Soleil et le Temps qui n'existe plus dans la cellule. La mise en musique de quinze de ces poèmes sur un ton tantôt d'une 
élégie et tantôt d'une lamentation souligne leur contenu sémantique et évoque parfaitement l'état d'esprit du compositeur.

Ce même état d'esprit est exprimé dans deux chansons qui font partie du cycle Chansons de lutte écrites, en 1970, pendant l'emprisonnement de Theodorakis au camp d'Oropos. La

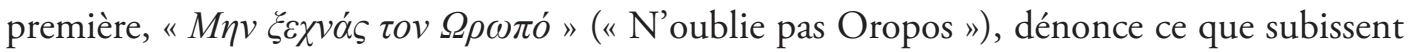
les prisonniers sous la Junte :

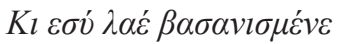

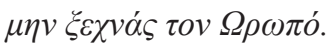

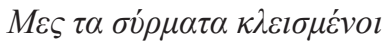 \\ $\mu \alpha \eta \kappa \alpha \rho \delta l \alpha \dot{\alpha} \mu \alpha \varsigma \pi \alpha \dot{v} \tau \tau \alpha o \rho \theta \dot{\eta}$

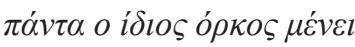

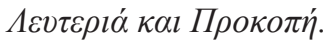

Et toi peuple torturé,

N'oublie pas Oropos.

Nous sommes enfermés dans les barbelés

Mais notre cœur reste toujours droit

Toujours notre serment demeure le même

Liberté et Progrès.

(Theodorakis 1997a, 233, v. 7-9)

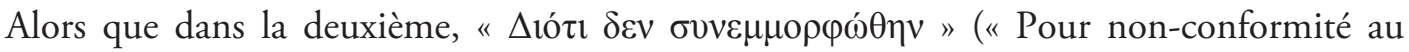
règlement "), le compositeur exprime, par la parole et la musique, sur un ton dérisoire, l'amertume qu'il éprouve dans sa prison :

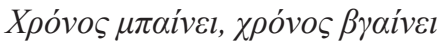

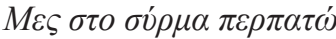 \\ $\Theta \alpha \pi \varepsilon \rho \dot{\sigma o v v v} \mu \alpha \dot{\rho} \rho \varepsilon \varsigma \mu \varepsilon \dot{\rho} \rho \varepsilon \varsigma$ \\ $\Delta i \chi \chi \omega \varsigma v \alpha \sigma \varepsilon \xi \alpha v \alpha \delta \omega ́$.

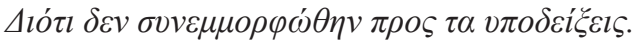 \\ Le temps s'en vient le temps s'en va \\ Je marche entre les barbelés \\ Bien des jours sombres s'écouleront \\ Avant de revoir ton visage \\ 'Pour non-conformité au règlement'. \\ (Theodorakis 1997a, 233, v. 5-7)
}

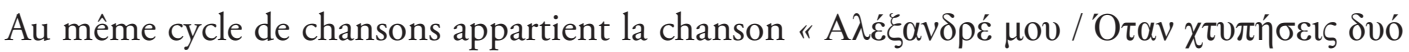
форе́́s" (" Mon Alexandre / Lorsque tu frapperas deux fois »). Ecrite en 1969, alors que 
Theodorakis était exilé à Zatouna en Arcadie, elle rend hommage à Alexandros Panagoulis Par ces vers, Theodorakis évoque l'esprit du vrai combattant qui ne craint ni la prison, ni les tortures, ni la mort, transmettant ainsi à toute les générations la valeur de ne pas trahir ses idées et d'y rester fidèle jusqu'à la mort :

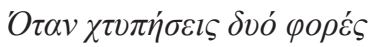

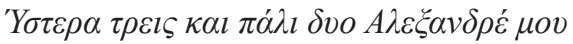

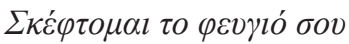

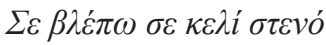

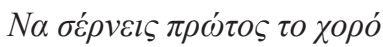

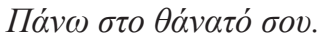

Lorsque tu frapperas deux fois

Et puis trois, et encore deux, Alexandre

Je pense à ta fuite

Je te vois dans ton étroite cellule

Mener le premier la danse

Sur ta mort.

(Theodorakis 1997a, 232, v. 27-33)

Les mélodies de ces chansons, composées respectivement sur le rythme des danses grecques traditionnelles, tsamikos et chasaposervikon, ainsi que sur le rythme de la marche, sont simples mais puissantes et soulignent de la meilleure manière le contenu sémantique des vers.

Le besoin éprouvé par Theodorakis d'inciter à la lutte pour les grandes valeurs humanistes tout en rendant hommage à ceux qui ont donné leur vie pour elles, est constant tout au long de sa vie. Ainsi, pendant les trente années du Nouveau Régime qui sont pour la Grèce une période de prospérité plutôt sereine, Theodorakis, retournant aux sources, se concentre de nouveau davantage sur la création de musique savante et compose, entre autres, des symphonies, des œuvres pour chœur et des opéras.

Si les œuvres mentionnées ci-dessus ont vu le jour lors des périodes politiques obscures où Theodorakis et ses idées étaient poursuivis et ont par conséquent connu la censure, l'œuvre

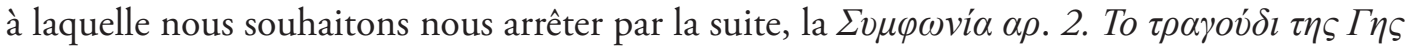
(Symphonie n. 2. Le chant de la Terre) pour orchestre, piano concertante et voix d'enfants, est créée en dehors des frontières grecques, suite à une nouvelle forme de censure. L'œuvre est composée en 1980-81, à Paris où Theodorakis trouve, encore une fois, refuge. Cette fois, il s'y auto-exile afin d'éviter l'isolement que les " puissants » du monde du spectacle en Grèce lui imposent comme il ne sert pas leurs intérêts (Stiga 2006, 218).

Au moment de la création de la Symphonie n. 2. Le chant de la Terre, Theodorakis réalise qu'il a commencé à composer cette œuvre dans les années cinquante puisqu'elle comprend du matériel musical issu de sa $\Sigma o v i \tau \alpha \alpha \rho .1$ (Suite n. 1), composée en 1954-1955, et créée par l'Orchestre Symphonique d'Athènes en 1955 avec comme soliste Jean Vigot, ainsi que 


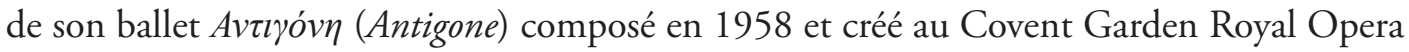
en 1959. L'œuvre est " une fresque sonore divisée en quatre parties-tableaux; chacun d'entre eux puise dans l'autre, tout en étant tous liés entre eux » (Theodorakis 1997b, 228). Dans les deux premières parties, l'œuvre exprime les traumatismes de la Seconde Guerre Mondiale et de la Guerre Civile Grecque qui étaient encore frais dans l'esprit du compositeur ainsi que l'angoisse provoquée par le nazisme, les camps de concentration et la bombe nucléaire. Dans

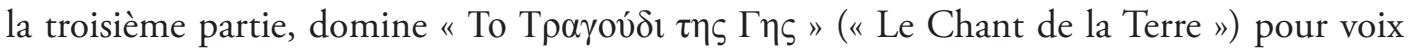
d'enfants sur les propres vers du compositeur :

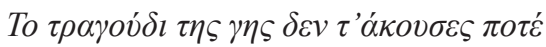

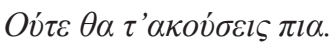

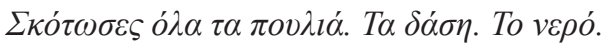

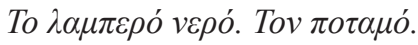

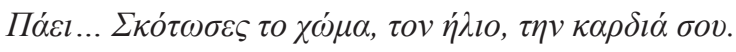

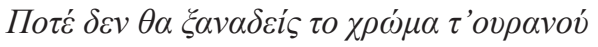

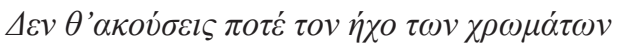

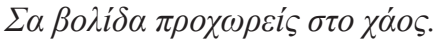

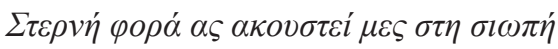

To $\tau \rho \alpha \gamma o v ́ \delta$ $\tau \eta \varsigma \gamma \eta \varsigma$.

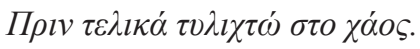

'Ev $\alpha<\gamma \varepsilon l \alpha \dot{\alpha} \sigma o v>\theta \alpha \pi \omega \sigma \tau \eta \zeta \omega \dot{\eta} \ldots$

Le chant de la terre tu ne l'as jamais entendu

Et tu ne l'entendras pas non plus.

Tu as tué tous les enfants. Les forêts. L'eau.

L'eau brillante. Le fleuve.

Il s'en va... Tu as tué la terre, le soleil, ton cœur.

Tu ne verras plus jamais la couleur du ciel.

Tu n'entendras plus jamais le son des couleurs.

Comme un bolide tu avances dans le chaos.

Que le chant de la terre soit entendu pour la dernière fois dans le silence.

Avant que je sois enveloppé par le chaos

C'est juste un « salut ` que je dirai à la vie...

(Theodorakis 1997b, 229)

Comme Theodorakis lui-même commente :

[...] le message semble pessimiste. Mais dans l'ensemble de l'économie de l'œuvre il résonne plutôt comme un cri de désespoir, peut-être pour faire peur, pour mobiliser même si ce n'est que pour cinq minutes, avant le chaos que les mouvements internationaux ont rendu sans aucun doute visible [...]. (Theodorakis 1997b, 228) 


\section{En guise de conclusion}

Poésie et Musique, Musique et Poésie, ceux sont là les deux armes principales de Theodorakis pour se délivrer, pour répandre ses idées humanistes, pour mobiliser les masses, pour exprimer aussi bien "le murmure de l'ermite que la clameur du monde " (Kampanellis 1996, 38). Et même si l'on pense souvent que le compositeur est le meilleur interprète de ses œuvres, même si les interprétations des chansons de Theodorakis par le compositeur luimême sont caractéristiques et pleines d'enthousiasme et de passion, ses œuvres n'auraient pu se répandre dans le monde entier sans ses interprètes dévoués qui, depuis plus de cinquante ans, parcourent le monde et donnent à ces mélodies magnifiques, corps et âme. De Maria Farantouri - muse de Theodorakis - à Nena Venetsanou, de Grigoris Bithikotis à Petros Pandis, de Manolis Mitsias à Dimitris Bassis et Petros Gaitanos, d'Edith Piaf aux Beatles, de Milva à Arja Sajionmaa etc. Tous ces artistes ainsi que les musiciens grecs et étrangers, membres des orchestres populaires et des orchestres symphoniques, ont joué un rôle déterminant dans la transformation de la musique theodorakienne en arme pacifique, pour la lutte consacrée à la réconciliation des peuples, à la Démocratie, à la Paix mondiale.

Pour conclure de manière poétique ce bref périple entre les mots et les mélodies de Mikis Theodorakis, nous laisserons la parole à la poétesse chinoise Xu Kai (SIOY KAI) qui écrit en s’adressant à Theodorakis :

Ave Mikis !

Je ne t'ai jamais vu

Mais ta mélodie

Bouleverse mon cœur.

Je demande au ciel,

Pourquoi?

Il me répond

Parce que tu es son fils,

Ta mélodie émane du ciel !

Je ne t'ai jamais vu

Mais ta mélodie

Bouleverse mon cœur.

Je demande à la terre,

Pourquoi ?

Elle me répond

Parce que tu es son fils,

Ta force émane de la terre! 
Je ne t'ai jamais vu

Mais ta mélodie

Bouleverse mon cœur.

Je demande au peuple,

Pourquoi ?

Il me répond

Parce que tu es son fils,

Ton âme émane du peuple !

(Logothetis 2004, 18)

\section{Notes}

1 Kalliopi Stigka (ou Stiga) est musicologue et professeure d'Éducation Musicale. Directrice du $\sigma^{\mathrm{e}}$ Collège du Pirée (Grèce).

2 Makronissos est une île cycladique toute sèche proche de la côte Est d'Attique, transformée en prison/en exil pour les communistes pendant la Guerre Civile Grecque.

3 Le mot $Y \pi \varepsilon \rho \mu \alpha ́ \alpha \omega$ (En hommage à la Vierge Combattante), appartient à l'hymne byzantin que les Grecs chantaient le jour de la prise de Constantinople par les Turcs en 1453 ; il s'agit d'un hymne consacré à la Vierge-Marie où ses ouailles lui promettaient de reconquérir Constantinople ainsi que l'Église de Sainte- Sophie qui était tombée aux mains des hétérodoxes.

4 Alexandros Panagoulis (1939-1976) était le lieutenant antifasciste qui a tenté d'assassiner le dictateur Georgios Papadopoulos. Sa tentative a échoué, il a été arrêté et torturé. Après la chute de la Junte, il a été élu député de l'Union de Gauche Démocratique et il a poursuivi, en tant que député, la chasse aux hommes politiques qui avaient collaboré avec le régime dictatorial. Il est mort le $1^{\text {er }}$ mai 1976, dans un accident de voiture, à la suite d'une nouvelle course poursuite, qui n'était pas la première, traqué par des hommes de la Droite.

\section{Bibliographie}

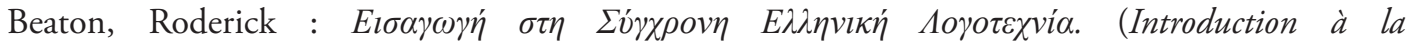
Littérature Grecque Moderne). Athènes : Nepheli, 1996.

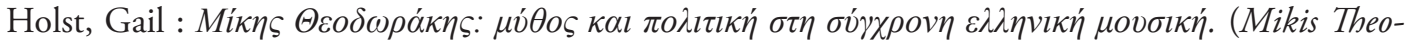
dorakis: Légende et politique de la musique grecque contemporaine). Athènes : Andromeda, 1980.

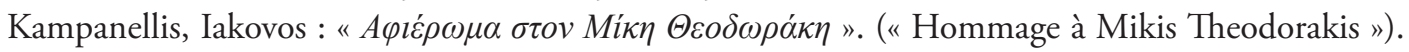

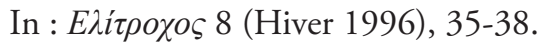

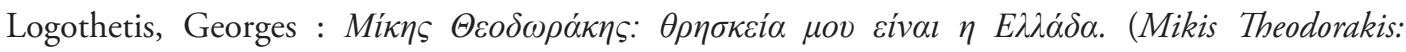
ma religion c'est la Grèce). Athènes: Agkyra, 2004.

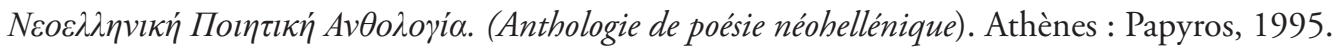




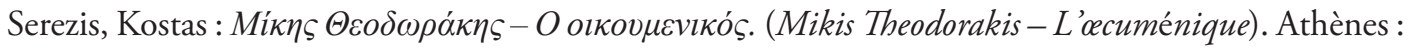
Kastaniotis, 2002.

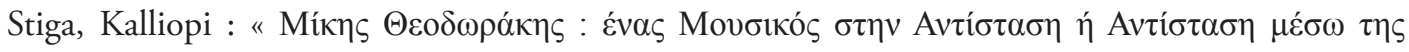
Моvбıкń » ("Mikis Theodorakis : un musicien dans la Résistance ou Résistance à travers la musique ?»). Conférence non publiée, réalisée le 25 mai 2002, dans le cadre des festivités organisées par la Municipalité de Karpenisi Evritanias pour l'Anniversaire de la Réunion du Conseil National par la P.E.E.A. le 27 mai 1944, à Koryschades à Evritania.

Stiga, Kalliopi : Mikis Theodorakis : le chantre du rapprochement de la musique savante et de la musique populaire. Thèse de Doctorat. Université Lumière Lyon II 2006.

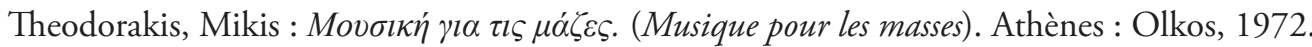

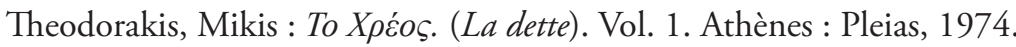

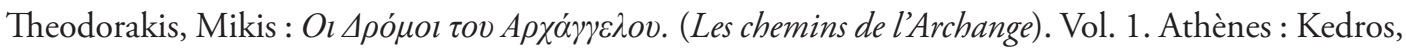
1986.

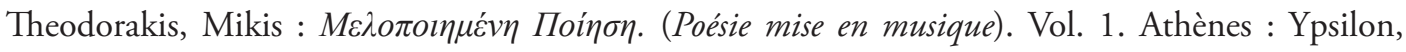
1997. [Theodorakis 1997a]

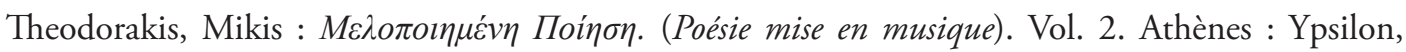
1997. [Theodorakis 1997b]

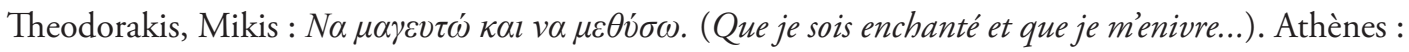
Livanis, 2000.

\section{Discographie}

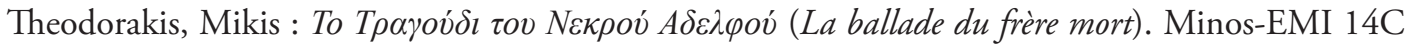
045 702072, 1960-1962 (CD).

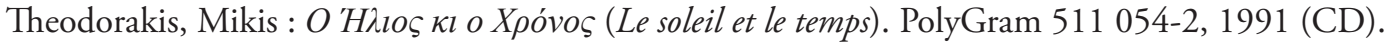

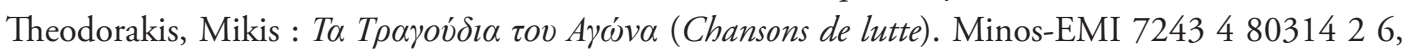
1992 (CD).

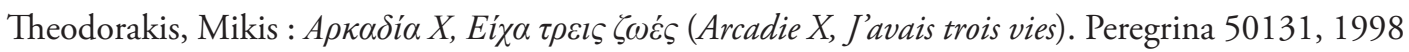
(CD).

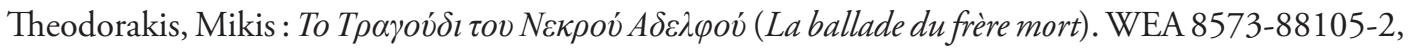
2001 (CD).

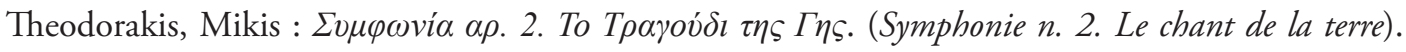
LEGEND 220 1155252, 2007 (CD). 